\title{
EXTENDING MULTIPLIERS OF THE FOURIER ALGEBRA FROM A SUBGROUP
}

\author{
MICHAEL BRANNAN AND BRIAN FORREST \\ (Communicated by Marius Junge)
}

\begin{abstract}
In this paper, we consider various extension problems associated with elements in the closure with respect to either the multiplier norm or the completely bounded multiplier norm of the Fourier algebra of a locally compact group. In particular, we show that it is not always possible to extend an element in the closure with respect to the multiplier norm of the Fourier algebra of the free group on two generators to a multiplier of the Fourier algebra of $S L(2, \mathbb{R})$.
\end{abstract}

\section{INTRODUCTION}

Let $G$ be a locally compact group. We let $A(G)$ and $B(G)$ denote the Fourier and Fourier-Stieltjes algebras of $G$. These Banach algebras of continuous functions on $G$, introduced by Eymard in [6], are central objects in non-commutative harmonic analysis.

A multiplier of $A(G)$ is a (necessarily bounded and continuous) function $v: G \rightarrow$ $\mathbb{C}$ such that $v A(G) \subseteq A(G)$. For each multiplier $v$ of $A(G)$, the linear operator $M_{v}$ on $A(G)$ defined by $M_{v}(u)=v u$ for each $u \in A(G)$ is bounded via the Closed Graph Theorem. The multiplier algebra of $A(G)$ is the closed subalgebra

$$
M A(G):=\left\{M_{v}: v \text { is a multiplier of } A(G)\right\}
$$

of $B(A(G))$, where $B(A(G))$ denotes the algebra of all bounded linear operators from $A(G)$ to $A(G)$. Throughout this paper we will generally use $v$ in place of the operator $M_{v}$ and we will write $\|v\|_{M A(G)}$ to represent the norm of $M_{v}$ in $B(A(G))$.

Let $G$ be a locally compact group and let $V N(G)$ be its group von Neumann algebra. That is, $V N(G)$ is the von Neumann algebra generated by the left regular representation $\lambda_{G}: G \rightarrow \mathcal{U}\left(L^{2}(G)\right)$. The duality

$$
A(G)=V N(G)_{*}
$$

equips $A(G)$ with a natural operator space structure. With this operator space structure we can define the $c b$-multiplier algebra of $A(G)$ to be

$$
M_{c b} A(G):=C B(A(G)) \cap M A(G),
$$

where $C B(A(G))$ denotes the (quantized) Banach algebra of all completely bounded linear maps from $A(G)$ into itself. We let $\|v\|_{M_{c b} A(G)}$ denote the $c b$-norm of the

Received by the editors April 13, 2011 and, in revised form, April 1, 2012.

1991 Mathematics Subject Classification. Primary 43A30, 43A22; Secondary 46L07, 22D25, 22D10.

Key words and phrases. Fourier algebra, multipliers, completely bounded multipliers, locally compact group. 
operator $M_{v}$. It is well known that $M_{c b} A(G)$ is a closed subalgebra of $C B(A(G))$ and is thus a (quantized) Banach algebra with respect to the norm $\|\cdot\|_{M_{c b} A(G)}$.

It is known that in general,

$$
A(G) \subseteq B(G) \subseteq M_{c b} A(G) \subseteq M A(G)
$$

and that for $v \in B(G)$,

$$
\|v\|_{B(G)} \geq\|v\|_{M_{c b} A(G)} \geq\|v\|_{M A(G)} .
$$

Moreover, if $v \in A(G)$, then

$$
\|v\|_{A(G)}=\|v\|_{B(G)} .
$$

In case $G$ is an amenable group, we have

$$
B(G)=M_{c b} A(G)=M A(G)
$$

and

for any $v \in B(G)$.

$$
\|v\|_{B(G)}=\|v\|_{M_{c b} A(G)}=\|v\|_{M A(G)}
$$

In [7, the first author introduced the following algebra, which was denoted in that paper by $A_{M_{0}}(G)$ :

Definition 1.1. Given a locally compact group $G$, let

$$
A_{c b}(G) \stackrel{\text { def }}{=} \overline{A(G)}\|\cdot\|_{M_{c b} A(G)} \subseteq M_{c b} A(G) .
$$

Clearly, if $G$ is amenable, then $A(G)=A_{c b}(G)$ and the norms agree. However, in general if $G$ is non-amenable, then $A(G) \subsetneq A_{c b}(G)$. Moreover, it can be shown that for a large class of locally compact groups which contains many interesting non-amenable groups, including the free group on two generators $\mathbb{F}_{2}$ and $S L(2, \mathbb{R}), A_{c b}(G)$ behaves in much the same manner as does the Fourier algebra of an amenable group [4, 8]. For this class, the so-called weakly amenable groups, $A_{c b}(G)$ has proven to be a rather interesting object to study.

In this paper, we will also be interested in multipliers of $A(G)$ that may or may not be completely bounded but can nonetheless be approximated by elements of $A(G)$. This leads us to the following algebras:

Definition 1.2. Given a locally compact group $G$, let

$$
A_{M}(G) \stackrel{\text { def }}{=} \overline{A(G)}\|\cdot\|_{M A(G)} \subseteq M A(G) .
$$

As we have seen, if $G$ is amenable, then $A(G)=A_{c b}(G)=A_{M}(G)$ with equality holding for the various norms. Moreover, in [14 Losert has shown that $G$ is amenable if and only if $A(G)=A_{M}(G)$. In fact, Losert showed that $G$ is amenable whenever the $\|\cdot\|_{B(G)}$ and the $\|\cdot\|_{M A(G)}$ norms are equivalent on $A(G)$.

Typically, we have

$$
A(G) \subset A_{c b}(G) \subset A_{M}(G) .
$$

Since it is well known that $M_{c b}(A(G)) \subsetneq M(A(G))$ for many non-amenable groups, it would be reasonable to speculate that for non-amenable groups the second inclusion is proper. While this was recently shown in 2] to be the case for the group $\mathbb{F}_{2}$, in what can only be described as a remarkable result, Losert has shown that for the group $S L(2, \mathbb{R})$, we have $M_{c b} A(S L(2, \mathbb{R}))=M A(S L(2, \mathbb{R}))$ and hence that

$$
A(S L(2, \mathbb{R})) \subsetneq A_{c b}(S L(2, \mathbb{R}))=A_{M}(S L(2, \mathbb{R})) .
$$




\section{Some General RESUlts on RESTRICTIONS AND EXTENSIONS OF COMPLETELY BOUNDED MULTIPLIERS}

Let $G$ be a locally compact group and let $\mathcal{A}(G)$ be any of the algebras $A(G)$, $B(G), M A(G), M_{c b} A(G), A_{M}(G)$, or $A_{c b}(G)$. We denote the left translation operator on $\mathcal{A}(G)$ by $g \in G$ by $L_{g}$ (i.e. $\left(L_{g} \varphi\right)(x)=\varphi\left(g^{-1} x\right)$ ).

Let $H \leq G$ be a closed subgroup. We will denote by $\left.\mathcal{A}(G)\right|_{H}$ the space of all restrictions of elements of $\mathcal{A}(G)$ to $H$. In each case, it is known that

$$
\left.\mathcal{A}(G)\right|_{H} \subseteq \mathcal{A}(H)
$$

and that the restriction map $R: \mathcal{A}(G) \rightarrow \mathcal{A}(H)$ given by

$$
R(u)=\left.u\right|_{H}
$$

is contractive.

A natural question arises:

Question 2.1. For which pairs $H \leq G$ is the map $R: \mathcal{A}(G) \rightarrow \mathcal{A}(H)$ surjective? Equivalently, for which pairs $H \leq G$ will it be that every element in $\mathcal{A}(H)$ extends to an element in $\mathcal{A}(H)$ ?

It is well known that in general $\left.B(G)\right|_{H} \subsetneq B(H)$ (see for example [5, pg. 92]). Since this can happen even when $G$ is amenable, we do not expect the restriction mapping to be surjective in general for either $M A(G)$ or $M_{c b} A(G)$.

In stark contrast, Herz [1] has shown that for any closed subgroup $H$ of any locally compact group $G$,

$$
\left.A(G)\right|_{H}=A(H) .
$$

Herz's result (which we refer to as Herz's restriction theorem) has proved to be a powerful tool in the study of Fourier algebra. Its usefulness leads us to ask specifically:

Question 2.2. If $\mathcal{A}(G)$ is either $A_{M}(G)$ or $A_{c b}(G)$, does $\left.\mathcal{A}(G)\right|_{H}=\mathcal{A}(H)$ ?

Here it is natural to focus our attention on the case where $H$ is non-amenable, since if $H$ is amenable, $\mathcal{A}(H)=A(H)$ and Herz's restriction theorem establishes the result in the affirmative. In the general case, since $A(H)$ is always dense in $\mathcal{A}(H)$, if we could show that the restriction map $R$ has closed range, then we would be done.

Now, while we have noted that $\left.B(G)\right|_{H} \subsetneq B(H)$ in many situations, we at least know that the range of the restriction map is closed. This statement may well be part of the folklore. A similar result was proved by Ghandehari [9, Lemma 3.2.6] for the algebra $B_{0}(G)=B(G) \cap C_{0}(G)$. We include the short proof for completeness.

Proposition 2.3. For any closed subgroup $H \leq G,\left.B(G)\right|_{H}$ is a closed subalgebra of $B(H)$.

Proof. Let $\pi: G \rightarrow \mathcal{U}\left(\mathcal{H}_{\pi}\right)$ be any weakly continuous unitary representation of $G$. Then we denote by $A_{\pi}(G)$ the $\|\cdot\|_{B(G)}$-closed linear span of the coefficient functions $\left\{g \mapsto\langle\pi(g) \xi \mid \eta\rangle: \xi, \eta \in \mathcal{H}_{\pi}\right\}[1]$.

Let $\omega: G \rightarrow \mathcal{U}\left(\mathcal{H}_{\omega}\right)$ denote the universal unitary representation of $G$. Then $B(G)=\left\{g \mapsto\langle\omega(g) \xi \mid \eta\rangle: \xi, \eta \in \mathcal{H}_{\omega}\right\}$. On the other hand, we have $\left.B(G)\right|_{H}=\{h \mapsto$ $\left.\left\langle\left.\omega\right|_{H}(h) \xi \mid \eta\right\rangle: \xi, \eta \in \mathcal{H}_{\omega}\right\} \subseteq A_{\left.\omega\right|_{H}}(H)$, where $\left.\omega\right|_{H}: H \rightarrow \mathcal{U}\left(\mathcal{H}_{\omega}\right)$ is the restricted 
representation. Since $A_{\left.\omega\right|_{H}}(H)$ is by definition norm closed, then $\overline{\left.B(G)\right|_{H}} \|^{\|\cdot\|_{B(H)}} \subseteq$ $A_{\left.\omega\right|_{H}}(H)$. On the other hand we have $\left.A_{\left.\omega\right|_{H}}(H) \subseteq B(G)\right|_{H}$. To see this, let $v \in A_{\left.\omega\right|_{H}}(H)$. Then there exist $\left.\left\{\xi_{i}\right\}_{i=1}\right|^{\infty},\left\{\eta_{i}\right\}_{i=1}^{\infty} \subset \mathcal{H}_{\omega}$ such that $\sum_{i=1}^{\infty}\left\|\xi_{i}\right\|^{2}<$ $\infty, \sum_{i=1}^{\infty}\left\|\xi_{i}\right\|^{2}<\infty$, and

$$
v=\sum_{i=1}^{\infty}\left\langle\left.\omega\right|_{H}(\cdot) \xi_{i} \mid \eta_{i}\right\rangle
$$

Clearly

$$
u=\sum_{i=1}^{\infty}\left\langle\omega(\cdot) \xi_{i} \mid \eta_{i}\right\rangle \in A_{\omega}(G)=B(G)
$$

satisfies $\left.u\right|_{H}=v$.

Remark 2.4. The proof above can be clearly modified to show that for any weakly continuous unitary representation $\pi$ of $G$, we have

$$
\left.A_{\pi}(G)\right|_{H}=A_{\left.\pi\right|_{H}}(G) .
$$

In particular, if $\lambda_{G}$ is the left regular repreesntation of $G$, then Herz's theorem can be interpreted as

$$
\left.A(G)\right|_{H}=\left.A_{\lambda_{G}}(G)\right|_{H}=A_{\left.\lambda_{G}\right|_{H}}(G)=A(H) .
$$

Proposition 2.3 naturally leads to the analogous question for multipliers.

Question 2.5. Let $H \leq G$ be closed. Is the restriction algebra $\left.M_{c b} A(G)\right|_{H}$ always closed in $M_{c b} A(H)$ ? Is $\left.M A(G)\right|_{H}$ always closed in $M A(H)$ ?

Before going on we wish to remark that in the case that $H$ is an open subgroup of $G$, it is a relatively straightforward exercise to show that the answer to the previous question is yes for both the multipliers and the completely bounded multipliers respectively. Indeed if $u \in M_{c b} A(H)$, then it is easy to show that

$$
v(x):= \begin{cases}u(x) & \text { if } x \in H \\ 0 & \text { if } x \notin H\end{cases}
$$

is in $M_{c b} A(H)$. A similar statement can be made if $u \in M A(H)$. It follows that if $H$ is open, then in each case the restriction map is a surjection.

\section{3. $[S I N]_{H}$ GROUPS}

Let $G$ be a locally compact group and $H \leq G$ a closed subgroup. We say that $G \in[S I N]_{H}$ if there exists an open neighbourhood base $\{V\}_{V \in \mathcal{V}}$ of the identity in $e \in G$ which is invariant under inner automorphisms by $H$. In this section, we show that for $G \in[S I N]_{H}$, we can provide partial answers to the above questions for cb multipliers. In particular, we prove a version of Herz's restriction theorem for the algebra $A_{c b}(G)$. Our main tool is the following construction due to Haagerup and Kraus [10.

Theorem 3.1 ([10, Lemma 1.16]). Let $H$ be a closed subgroup of a locally compact group $G$ and suppose that the Haar modular functions satisfy the relation $\Delta_{H}=$ $\left.\Delta_{G}\right|_{H}$. Fix $\xi \in C_{c}(G)$. Define a linear map $\Phi_{\xi}: C_{b}(H) \rightarrow C_{b}(G)$ by the equation

$$
\Phi_{\xi}(\varphi)=\xi *(\varphi d h) * \tilde{\xi}, \quad\left(\varphi \in C_{b}(H)\right),
$$


where $\tilde{\xi}(g)=\overline{\xi\left(g^{-1}\right)}$. Then $\Phi_{\xi}\left(M_{c b} A(H)\right) \subseteq M_{c b} A(G)$ and

$$
\left\|\Phi_{\xi}\right\|_{M_{c b} A(H) \rightarrow M_{c b} A(G)} \leq \int_{G / H}\left(\int_{H} \xi(g h) d h\right)^{2} d(g H),
$$

where $d(g H)$ is the left translation-invariant measure on $G / H$ induced by Haar measure $d g$ in $G$, and normalized so that

$$
\int_{G} f(g) d g=\int_{G / H} \int_{H} f(g h) d h d(g H) \quad\left(f \in C_{c}(G)\right) .
$$

Our next result, the main result of this section, is inspired by the work of Cowling and Rodway [5] on extending elements of $B(H)$ to $B(G)$ when $G$ is a SIN-group and $H \leq G$ is closed.

Theorem 3.2. Let $G \in[S I N]_{H}$, and suppose $\varphi \in M_{c b} A(H)$ has the property that the map

$$
\begin{aligned}
H & \rightarrow M_{c b} A(H), \\
h & \mapsto L_{h} \varphi
\end{aligned}
$$

is continuous. Then $\left.\varphi \in M_{c b} A(G)\right|_{H}$. Moreover, for any $\epsilon>0$, there exists $u \in$ $M_{c b} A(G)$ such that $\left.u\right|_{H}=\varphi$ and $\|u\|_{M_{c b} A(G)} \leq(1+\epsilon)\|\varphi\|_{M_{c b} A(H)}$.

Remark 3.3. Observe that if $H$ is discrete and $G \in[S I N]_{H}$, then every $\varphi \in$ $M_{c b} A(H)$ trivially satisfies the hypothesis of Theorem 3.2 so $M_{c b} A(H)=$ $\left.M_{c b} A(G)\right|_{H}$ in this case. When $H$ is not discrete or amenable, it is unknown whether or not every element of $M_{c b} A(H)$ satisfies the translation-continuity condition of this theorem. Other examples of $\varphi \in M_{c b} A(H)$ which do satisfy the hypothesis of Theorem 3.2 are given by coefficient functions of uniformly bounded (not necessarily unitary) representations of $H$ and elements which belong to the closure $\overline{B(H)}\|\cdot\|_{M_{c b} A(H)} \subseteq M_{c b} A(H)$.

Proof of Theorem 3.2. Fix $\varphi \in M_{c b} A(H)$ satisfying the above hypothesis. As in the proof of the open mapping theorem (see for example [17, Theorem 5.9]), it suffices to prove that for any $\epsilon>0$ there exists $u \in M_{c b} A(G)$ with

$$
\|u\|_{M_{c b} A(G)} \leq\|\varphi\|_{M_{c b} A(H)}
$$

and

$$
\left\|\left.u\right|_{H}-\varphi\right\|_{M_{c b} A(H)}<\epsilon .
$$

Let $V$ be an open neighbourhood of the identity $e \in G$ such that

$$
\left\|L_{h^{-1}} \varphi-\varphi\right\|_{M_{c b} A(H)}<\epsilon \quad\left(h \in V^{-1} V \cap H\right) .
$$

Let $0 \leq \xi \in C_{c}(G)$ be a function which is $H$-central (i.e. $\xi\left(h x h^{-1}\right)=\xi(x)$ for all $h \in H, x \in G)$ and suppose $\operatorname{supp} \xi \subseteq V$. This is always possible, since $G \in[S I N]_{H}$. Finally normalize $\xi$ so that

$$
\int_{G / H}\left(\int_{H} \xi(g h) d h\right)^{2} d(g H)=1 .
$$

Let $u \in M_{c b} A(G)$ be defined by $u=\Phi_{\xi}(\varphi)$, where $\Phi_{\xi}$ is the map given in Theorem 3.1. Then

$$
\|u\|_{M_{c b} A(G)} \leq\|\varphi\|_{M_{c b} A(H)} \int_{G / H}\left(\int_{H} \xi(g h) d h\right)^{2} d(g H)=\|\varphi\|_{M_{c b} A(H)} .
$$


We now consider the restriction $\left.u\right|_{H} \in M_{c b} A(H)$. For $k \in H$ we have

$$
\begin{aligned}
u(k) & =(\xi *(\varphi d h) * \tilde{\xi})(k) \\
& =\int_{G} \int_{H} \xi(g) \varphi(h) \xi\left(k^{-1} g h\right) d h d g \\
& =\int_{G} \int_{H} \xi(g) \varphi(h) \xi\left(g h k^{-1}\right) d h d g \quad \text { (since } \xi \text { is } H \text {-central) } \\
& =\int_{G} \int_{H} \xi(g) \varphi(h k) \xi(g h) d h d g \quad \text { (since } H \text { is unimodular) } \\
& =\int_{G} \int_{H} \xi(g) \xi(g h)\left(L_{h^{-1}} \varphi\right)(k) d h d g .
\end{aligned}
$$

On the other hand, note that

$$
\begin{aligned}
& \int_{G} \int_{H} \xi(g) \xi(g h) d h d g \\
= & \int_{G / H} \int_{H} \int_{H} \xi\left(g h^{\prime}\right) \xi\left(g h^{\prime} h\right) d h d h^{\prime} d(g H) \\
= & \int_{G / H} \int_{H} \int_{H} \xi\left(g h^{\prime}\right) \xi(g h) d h d h^{\prime} d(g H) \\
= & \int_{G / H}\left(\int_{H} \xi(g h) d h\right)^{2} d(g H)=1 .
\end{aligned}
$$

Therefore, for all $k \in H$, we have

$$
u(k)-\varphi(k)=\int_{G} \int_{H} \xi(g) \xi(g h)\left[\left(L_{h^{-1}} \varphi\right)(k)-\varphi(k)\right] d h d g .
$$

Since the function

$$
G \times H \ni(g, h) \mapsto v(g) v(g h)
$$

is non-negative, continuous, and compactly supported, we can interpret the difference $\left.u\right|_{H}-\varphi$ as the vector valued integral

$$
\left.u\right|_{H}-\varphi=\int_{G} \int_{H} \xi(g) \xi(g h)\left[L_{h^{-1}} \varphi-\varphi\right] d h d g \in M_{c b} A(H) .
$$

Furthermore, we have the norm estimate

$$
\begin{aligned}
\left\|\left.u\right|_{H}-\varphi\right\|_{M_{c b} A(H)} & \leq \int_{G} \int_{H} \xi(g) \xi(g h)\left\|L_{h^{-1}} \varphi-\varphi\right\|_{M_{c b} A(H)} d h d g \\
& \leq\{(g, h) \in G \times H: v(g) v(g h) \neq 0\} \\
& \leq \sup _{\left\{g \in V, h \in V^{-1} V \cap H\right\}}\left\|L_{h^{-1}} \varphi-\varphi\right\|_{M_{c b} A(H)} \\
& <\epsilon .
\end{aligned}
$$

This completes the proof.

As a consequence of Theorem 3.2 , we get an analogue of Herz's restriction theorem for $A_{c b}(G)$ when $G \in[S I N]_{H}$.

Corollary 3.4. If $G \in[S I N]_{H}$, then the restriction map $R: A_{c b}(G) \rightarrow A_{c b}(H)$ is a completely contractive surjection. 
Proof. It is well known that the restriction map $R: M_{c b} A(G) \rightarrow M_{c b} A(H)$ is a complete contraction (see [18, Corollary 6.3]). We therefore only need to show that $R: A_{c b}(G) \rightarrow A_{c b}(H)$ is surjective.

Let $\varphi \in A_{c b}(H)$ and $\epsilon>0$ be arbitrary. We want to show that $\varphi \in R\left(A_{c b}(G)\right)$. Using the same open mapping theorem argument that was used at the start of the proof of Theorem 3.2, our problem reduces to finding $u \in A_{c b}(G)$ such that $\|u\|_{A_{c b}(G)} \leq\|\varphi\|_{A_{c b}(H)}$ and $\left\|\left.u\right|_{H}-\varphi\right\|_{A_{c b}(H)}<\epsilon$. Since $\varphi \in A_{c b}(H) \subseteq \overline{B(H)}^{\|\cdot\|_{M_{c b} A(H)}}$, $\varphi$ satisfies the hypothesis of Theorem 3.2 (see Remark 3.3). Let $u=\Phi_{\xi}(\varphi) \in$ $M_{c b} A(G)$ be the cb multiplier constructed in the proof of Theorem 3.2. Then $\|u\|_{M_{c b} A(G)} \leq\|\varphi\|_{A_{c b}(H)}$ and $\left\|\left.u\right|_{H}-\varphi\right\|_{A_{c b}(H)}<\epsilon$. So to complete the proof, we need to show that $u \in A_{c b}(G)$. This, however, is easy: using the density of $A(H) \cap C_{c}(H)$ in $A_{c b}(H)$, we can find a sequence $\left\{\varphi_{n}\right\}_{n \in \mathbb{N}} \subset A(H) \cap C_{c}(H)$ such that

$$
\lim _{n \rightarrow \infty}\left\|\varphi-\varphi_{n}\right\|_{M_{c b} A(H)}=0 .
$$

Since $\Phi_{\xi}: M_{c b} A(H) \rightarrow M_{c b} A(G)$ is a continuous map which evidently maps compactly supported functions to compactly supported functions, we have

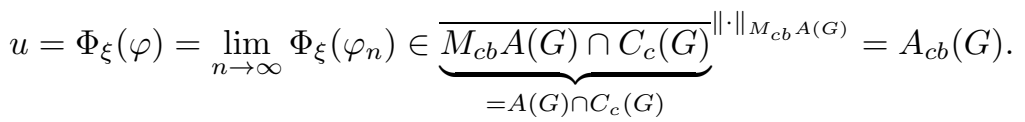

It turns out that when $G \in[S I N]_{H}$ and $H$ is a discrete subgroup, we can improve on Theorem 3.2 and Corollary 3.4 a bit:

Theorem 3.5. Suppose that $G \in[S I N]_{H}$ and that $H$ is a discrete subgroup. Then there exists an isometry $\Gamma: M_{c b} A(H) \rightarrow M_{c b} A(G)$ which maps $A_{c b}(H)$ into $A_{c b}(G)$ and is a right inverse for the restriction map. That is, $\left.\Gamma \varphi\right|_{H}=\varphi$ for all $\varphi \in$ $M_{c b} A(H)$.

An immediate consequence of this theorem is the following:

Corollary 3.6. Let $H \leq G$ be as in Theorem 3.5, and let $\mathcal{A}(G)$ (resp. $\mathcal{A}(H))$ be either the algebra $M_{c b} A(G)$ or $A_{c b}(G)$ (resp. $M_{c b} A(H)$ or $A_{c b}(H)$ ). Let $I_{G}(H)$ denote the ideal of functions $\psi \in \mathcal{A}(G)$, which vanish on $H$. Then $I_{G}(H)$ is complemented in $\mathcal{A}(G)$. Furthermore, this is also true with $H$ replaced by any set $E \subseteq H$ for which $I_{H}(E)$, the ideal of those functions in $\mathcal{A}(H)$ vanishing on $E$, is complemented in $\mathcal{A}(H)$.

Remark 3.7. We suspect that the above results may well be true with "complete isometry" replacing isometry and "completely complemented" replacing complemented. However, we are at this time unable to prove this assertion.

It is also worth noting that the previous two results are new even if $G$ is amenable.

We will now prove Theorem 3.5 .

Proof. Choose an open symmetric neighborhood $V$ of the identity $e \in G$ with compact closure such that $H \cap V=H \cap V^{2}=\{e\}$. This can always be done because $H$ is a discrete subgroup of $G$. Next, let $0 \leq \xi \in C_{c}(G)$ be a function which is $H$-central (i.e. $\xi\left(h x h^{-1}\right)=\xi(x)$ for all $h \in H, x \in G$ ) and suppose $\operatorname{supp} \xi \subseteq V$. This is possible because $G \in[S I N]_{H}$. 
Let $u=\xi * \tilde{\xi} \in A(G) \cap C_{c}(G)$ and normalize $\xi$ so that $u(e)=\|\xi\|_{L^{2}(G)}^{2}=1$. Note that $\operatorname{supp}(u) \subseteq V^{2}$. Define

$$
\Gamma: \ell^{\infty}(H) \rightarrow C_{b}(G)
$$

by the equation

$$
(\Gamma \varphi)(x)=\sum_{h \in H} \varphi(h) u\left(h^{-1} x\right) .
$$

Then $\Gamma$ maps finitely supported functions to compactly supported functions, and $\left.\Gamma \varphi\right|_{H}=\varphi$ for all $\varphi \in \ell^{\infty}(H)$. To verify this last statement, note that for any $h, k \in H, u=\xi * \breve{\xi}$ has been chosen so that $u\left(h^{-1} k\right)=\delta_{h, k}$. Therefore

$$
(\Gamma \varphi)(k)=\sum_{h \in H} \varphi(h) u\left(h^{-1} k\right)=\varphi(k) \quad\left(\varphi \in \ell^{\infty}(H), k \in H\right) .
$$

Next we observe that $\Gamma=\Phi_{\xi}: M_{c b} A(H) \rightarrow M_{c b} A(G)$.

For $\varphi \in M_{c b} A(H)$ and $x \in G$ we have

$$
\begin{aligned}
\Gamma \varphi(x) & =\sum_{h \in H} \varphi(h) u\left(h^{-1} x\right) \\
& =\int_{G} \sum_{h \in H} \xi(g) \varphi(h) \xi\left(x^{-1} h g\right) d g \\
& =\int_{G} \sum_{h \in H} \xi\left(h^{-1} g\right) \varphi(h) \xi\left(x^{-1} g\right) d g \\
& =\int_{G} \sum_{h \in H} \xi\left(g h^{-1}\right) \varphi(h) \xi\left(x^{-1} g\right) d g \\
& =\int_{G} \sum_{h \in H} \xi(g) \varphi(h) \xi\left(x^{-1} g h\right) d g \\
& =\int_{G} \sum_{h \in H} \xi(g) \varphi(h) \tilde{\xi}\left(h^{-1} g^{-1} x\right) d g \\
& =\int_{G} \xi(g)[(\varphi d h) * \tilde{\xi}]\left(g^{-1} x\right) d g \\
& =[\xi *(\varphi d h) * \tilde{\xi}](x)=\left[\Phi_{\xi}(\varphi)\right](x) .
\end{aligned}
$$

So $\Gamma=\Phi_{\xi}: M_{c b} A(H) \rightarrow M_{c b} A(G)$ and by Theorem 3.1 .

$$
\begin{aligned}
& \|\Gamma\|=\left\|\Phi_{\xi}\right\| \\
\leq & \int_{G / H}\left(\sum_{h \in H} \xi(g h)\right)^{2} d(g H) \\
= & \int_{G / H} \sum_{h, h^{\prime} \in H} \xi(g h) \xi\left(g h^{\prime}\right) d(g H) \\
= & \int_{G / H} \sum_{h \in H} \xi(g h)^{2} d(g H) \\
& \left(\text { since } g \mapsto \xi(g h) \text { and } g \mapsto \xi\left(g h^{\prime}\right) \text { have disjoint supports for } h \neq h^{\prime}\right) \\
= & \int_{G} \xi(g)^{2} d g=\|\xi\|_{L^{2}(G)}^{2}=1 .
\end{aligned}
$$


The fact that $\Gamma$ is an isometry now follows from the contractivity of $\Gamma$ and the fact that restriction to $H$ is a complete contraction from $M_{c b} A(G)$ to $M_{c b} A(H)$. Indeed, for $\varphi \in M_{c b} A(H)$,

$$
\|\Gamma \varphi\|_{M_{c b} A(G)} \leq\|\varphi\|_{M_{c b} A(H)}=\left\|\left.\Gamma \varphi\right|_{H}\right\|_{M_{c b} A(H)} \leq\|\Gamma \varphi\|_{M_{c b} A(G)} .
$$

\section{The GRoup $S L(2, \mathbb{R})$}

Now let's consider the case where $G$ is the connected Lie group $S L(2, \mathbb{R})$ and $H \leq G$ is a copy of the discrete subgroup $\mathbb{F}_{2}$. For example, we may take $H$ to be the discrete subgroup generated by the (algebraically free) pair of matrices

$$
g_{1}=\left(\begin{array}{ll}
1 & 2 \\
0 & 1
\end{array}\right), \quad g_{2}=\left(\begin{array}{ll}
1 & 0 \\
2 & 1
\end{array}\right) .
$$

Our main tool will be the following result due to Losert:

Theorem 4.1 ([15, Theorem]). For $G=S L(2, \mathbb{R})$, we have $M A(G)=M_{c b} A(G)$, and $A(G)$ is dense in $M A(G) \cap C_{0}(G)$ with respect to $\|\cdot\|_{M A(G)}$. For any $u \in$ $M A(G), \lambda=\lim _{x \rightarrow \infty} u(x)$ exists, $u-\lambda \in M A(G) \cap C_{0}(G)$, and $\|u\|_{M A(G)}=$ $\|u-\lambda\|_{M A(G)}+\lambda$.

In particular, this result says that $A_{M}(G)=A_{c b}(G)=M_{c b} A(G) \cap C_{0}(G)$ and

$$
M_{c b} A(G)=\mathbb{C} 1_{G} \oplus_{1} A_{c b}(G) .
$$

Losert's result immediately gives a negative answer to Question 2.2 for $A_{M}(G)$ and Question 2.5 for $M A(G)$ with this choice of $G$ and $H$. To see why this is the case, we first need the following proposition.

Proposition 4.2. For the free group on two generators $\mathbb{F}_{2}$, we have that

$$
A_{c b}\left(\mathbb{F}_{2}\right) \subsetneq A_{M}\left(\mathbb{F}_{2}\right) .
$$

Proof. Since $\|v\|_{M_{c b} A\left(\mathbb{F}_{2}\right)} \geq\|v\|_{M A\left(\mathbb{F}_{2}\right)}$, if $A_{c b}\left(\mathbb{F}_{2}\right)=A_{M}\left(\mathbb{F}_{2}\right)$, then it follows immediately that the two norms are equivalent on $A\left(\mathbb{F}_{2}\right)$. This is impossible since in [3. Theorem 6.3.3] the first author constructs a set $E \subset \mathbb{F}_{2}$ such that the ideal

$$
I(E)=\left\{u \in A\left(\mathbb{F}_{2}\right) \mid u(g)=0 \text { for all } g \in E\right\} \subset A\left(\mathbb{F}_{2}\right)
$$

has an approximate identity that is bounded in the $\|\cdot\|_{M A\left(\mathbb{F}_{2}\right)}$ norm but not in the $\|\cdot\|_{M_{c b} A\left(\mathbb{F}_{2}\right)}$ norm.

Theorem 4.3. The restriction map from $A_{M}(G)$ to $A_{M}(H)$ is not surjective, and $\left.M A(G)\right|_{H}$ is not closed in $M A(H)$.

Proof. Consider the first statement concerning restriction from $A_{M}(G)$ to $A_{M}(H)$. By Theorem 4.1. $A_{M}(G)=A_{c b}(G) \subset M_{c b} A(G)$. Since restriction from $G$ to $H$ induces a complete contraction from $M_{c b} A(G)$ into $M_{c b} A(H)$, we also have $\left.A_{M}(G)\right|_{H} \subseteq A_{c b}(H)$. On the other hand, for $H=\mathbb{F}_{2}$, we know that $A_{M}(H) \subsetneq$ $A_{c b}(H)$, so $\left.A_{M}(G)\right|_{H} \subsetneq A_{M}(H)$. This proves the first statement.

Now consider the restriction algebra $\left.M A(G)\right|_{H}$. Take any element

$$
\varphi \in A_{M}(H) \backslash M_{c b} A(H) .
$$

Then, by Theorem 4.1, $\left.M A(G)\right|_{H} \subseteq M_{c b} A(H)$, so

$$
\left.\varphi \notin M A(G)\right|_{H} \text {. }
$$


On the other hand, we claim that

$$
\varphi \in{\overline{\left.M A(G)\right|_{H}}}^{\|\cdot\|_{M A(H)}} .
$$

To see this, let $\left\{\varphi_{n}\right\}_{n \in \mathbb{N}} \subseteq A(H)$ be a sequence such that $\lim _{n \rightarrow \infty}\left\|\varphi_{n}-\varphi\right\|_{M A(H)} \rightarrow$ 0 . Then, by Herz's restriction theorem,

$$
\left.\left.\left\{\varphi_{n}\right\}_{n \in \mathbb{N}} \subseteq A(G)\right|_{H} \subseteq M A(G)\right|_{H} .
$$

Therefore $\varphi \in \overline{\left.M A(G)\right|_{H}}\|\cdot\|_{M A(H)}$.

On the level of cb multipliers, with the choice of $G$ and $H$ as above, the situation is quite different than it is for $[S I N]_{H}$ groups (cf. Theorem 3.5).

Theorem 4.4. The restriction map from $M_{c b} A(G)$ to $M_{c b} A(H)$ is not surjective.

Proof. Let $g_{1}, g_{2}$ be the free generators of $H=\mathbb{F}_{2}$. Then the set $E=\left\{g_{1}^{n} g_{2} g_{1}^{-n}\right\}_{n \in \mathbb{N}}$ $\subset H$ is algebraically free. It follows from the work of Leinert [13] (or more recently [16, Theorem 0.1]) that

$$
\left\{\varphi \in M_{c b} A(H): \operatorname{supp} \varphi \subseteq E\right\}=\ell^{\infty}(E),
$$

completely isomorphically.

Now choose any $\varphi \in \ell^{\infty}(E)$ such that $\lim _{n \rightarrow \infty} \varphi\left(g_{1}^{n} g_{2} g_{1}^{-n}\right)$ does not exist. (For example, take $\varphi\left(g_{1}^{n} g_{2} g_{1}^{-n}\right)=(-1)^{n}$.) We claim that there is no $u \in M_{c b} A(G)$ such that $\left.u\right|_{H}=\varphi$. Indeed, if such a $u$ existed, Theorem 4.1 would imply that

$$
\lim _{x \rightarrow \infty} u(x)=\lambda \in \mathbb{C} .
$$

But since

$$
g_{1}^{n} g_{2} g_{1}^{-n} \rightarrow \infty \quad(n \rightarrow \infty)
$$

this would mean that

$$
\lim _{n \rightarrow \infty} \varphi\left(g_{1}^{n} g_{2} g_{1}^{-n}\right)=\lim _{n \rightarrow \infty} u\left(g_{1}^{n} g_{2} g_{1}^{-n}\right)=\lambda,
$$

which is a contradiction.

Remark 4.5. At this point we do not know if $\left.M_{c b} A(S L(2, \mathbb{R}))\right|_{\mathbb{F}_{2}}$ is closed in $M_{c b} A\left(\mathbb{F}_{2}\right)$, and hence whether or not $A_{c b}\left(\mathbb{F}_{2}\right)=\left.A_{c b}(S L(2, \mathbb{R}))\right|_{\mathbb{F}_{2}}$.

We note that it may well be possible that the above equality does hold. One suggestive piece of evidence in this direction arises from the representation theorem for cb multipliers. Let $\xi \in L^{2}(G)$ be any unit vector, and let $\left(\omega, \mathcal{H}_{\omega}\right)$ be the universal unitary representation of $G$. Examining the proof of Jolissaint [12, we see that for $\varphi \in M_{c b} A(G)$, there exist bounded maps $V_{1}, V_{2}: L^{2}(G) \rightarrow \mathcal{H}_{\omega}$ such that

$$
\|\varphi\|_{M_{c b} A(G)}=\left\|V_{1}\right\|\left\|V_{2}\right\|
$$

and such that $\varphi$ is represented by the "twisted coefficient" function

$$
\varphi\left(y^{-1} x\right)=\left\langle\omega(x) V_{1} \lambda\left(x^{-1}\right) \xi \mid \omega(y) V_{2} \lambda\left(y^{-1}\right) \xi\right\rangle \quad(x, y \in G) .
$$

So any restriction $\left.\psi \in M_{c b} A(G)\right|_{H}$ will be a "twisted coefficient" associated to $\left.\omega\right|_{H}$. This is somewhat reminiscent of the situation for $B(G)$, though of course we are lacking anything as complete as Arsac's work on $B(G)$ [1] in our understanding of $M_{c b} A(G)$ to help us complete the argument. 


\section{REFERENCES}

[1] Gilbert Arsac, Sur l'espace de Banach engendré par les coefficients d'une représentation unitaire, Publ. Dép. Math. (Lyon) 13 (1976), no. 2, 1-101 (French). MR0444833 (56 \#3180)

[2] M. Brannan, B. Forrest and C. Zwarich, Multipliers, completely bounded multipliers, and ideals in the Fourier algebra. Preprint, 2011.

[3] M. Brannan, Operator spaces and ideals in Fourier algebras. Thesis, University of Waterloo, 2008.

[4] Michael Cowling and Uffe Haagerup, Completely bounded multipliers of the Fourier algebra of a simple Lie group of real rank one, Invent. Math. 96 (1989), no. 3, 507-549, DOI 10.1007/BF01393695. MR996553(90h:22008)

[5] Michael Cowling and Paul Rodway, Restrictions of certain function spaces to closed subgroups of locally compact groups, Pacific J. Math. 80 (1979), no. 1, 91-104. MR.534697 (80i:43008)

[6] Pierre Eymard, L'algèbre de Fourier d'un groupe localement compact, Bull. Soc. Math. France 92 (1964), 181-236 (French). MR0228628 (37 \#4208)

[7] Brian Forrest, Some Banach algebras without discontinuous derivations, Proc. Amer. Math. Soc. 114 (1992), no. 4, 965-970, DOI 10.2307/2159614. MR1068120 (92g:43001)

[8] Brian E. Forrest, Volker Runde, and Nico Spronk, Operator amenability of the Fourier algebra in the cb-multiplier norm, Canad. J. Math. 59 (2007), no. 5, 966-980, DOI 10.4153/CJM2007-041-9. MR2354398 (2008k:43005)

[9] M. Ghandehari, Harmonic analysis of Rajchman algebras. Thesis, University of Waterloo, 2010.

[10] Uffe Haagerup and Jon Kraus, Approximation properties for group $C^{*}$-algebras and group von Neumann algebras, Trans. Amer. Math. Soc. 344 (1994), no. 2, 667-699, DOI 10.2307/2154501. MR 1220905 (94k:22008)

[11] Carl Herz, Harmonic synthesis for subgroups, Ann. Inst. Fourier (Grenoble) 23 (1973), no. 3, 91-123 (English, with French summary). MR0355482 (50 \#7956)

[12] Paul Jolissaint, A characterization of completely bounded multipliers of Fourier algebras, Colloq. Math. 63 (1992), no. 2, 311-313. MR.1180643 (93j:43007)

[13] Michael Leinert, Faltungsoperatoren auf gewissen diskreten Gruppen, Studia Math. 52 (1974), 149-158 (German). MR0355480 (50 \#7954)

[14] Viktor Losert, Properties of the Fourier algebra that are equivalent to amenability, Proc. Amer. Math. Soc. 92 (1984), no. 3, 347-354, DOI 10.2307/2044833. MR759651 (86b:43010)

[15] V. Losert, On multipliers and completely bounded multipliers - the case $S L(2, \mathbb{R})$. Preprint, 2010.

[16] Gilles Pisier, Multipliers and lacunary sets in non-amenable groups, Amer. J. Math. 117 (1995), no. 2, 337-376, DOI 10.2307/2374918. MR.1323679(96e:46078)

[17] Walter Rudin, Real and complex analysis, 3rd ed., McGraw-Hill Book Co., New York, 1987. MR.924157 (88k:00002)

[18] Nico Spronk, Measurable Schur multipliers and completely bounded multipliers of the Fourier algebras, Proc. London Math. Soc. (3) 89 (2004), no. 1, 161-192, DOI 10.1112/S0024611504014650. MR2063663(2005b:22010)

Department of Mathematics and Statistics, Queen's University, 99 University Avenue, Kingston, ON, Canada, K7L 3N6

E-mail address: mbrannan@mast.queensu.ca

Department of Pure Mathematics, University of Waterloo, Waterloo, On, Canada, N2L $3 \mathrm{G} 1$

E-mail address: beforrest@uwaterloo.ca 